\title{
On controllability of timed continuous Petri nets
}

\author{
C. Renato Vázquez ${ }^{1}$, Antonio Ramírez ${ }^{2}$, Laura Recalde ${ }^{1}$, and Manuel Silva ${ }^{1 \star}$ \\ 1 Dep. de Informática e Ingeniería de Sistemas, Centro Politécnico Superior, \\ Universidad de Zaragoza, María de Luna 1, E-50018 Zaragoza, Spain \\ \{cvazquez, lrecalde, silva\}@unizar.es \\ 2 CINVESTAV-IPN Unidad Guadalajara, 45090 Guadalajara, Mexico \\ art@gdl. cinvestav. $\mathrm{mx}$
}

\begin{abstract}
Continuous Petri Nets is a subclass of hybrid models representing relaxed views of discrete events systems, in which timing may adopt different semantics. Even if no semantics is strictly superior, we proved in [1] that for an important subclass of models infinite servers semantics provides always a better approximation of the of the underlying discrete model than finite servers. This paper then concentrates on controllability under this semantics. First we propose a notion of controllability over subsets of the reachable polytope, and provide a necessary and sufficient condition for markings with no null elements (interior points); later the transformation of an arbitrary initial marking into an interior one is done. The technically more involved part of the paper is the extension of those results to the case in which some transitions are non controllable. An interesting point is that all characterisations are structural (i.e., depend only on the structure and firing speeds of the timed continuous net).
\end{abstract}

\section{Introduction}

Petri Nets constitute a well-known paradigm useful to model discrete event systems. In many practical cases, an enumeration approach has to be used to verify some properties of net models. Unfortunately, for highly marked systems, even for bounded, the reachability graph can be so large that many properties cannot be analyzed. This problem is known as the state explosion problem. Systems that frequently appear in practice, for instance in manufacturing, telecommunications, traffic or logistic, lead to Petri net models with many states. So, to analyze such systems fluidification has been proposed.

Fluidification constitutes a relaxation technique to study discrete systems through a "similar" but continuous model. Using fluid models, more analytical techniques can be used for the analysis of some interesting properties. In Petri Nets, fluidification has been introduced from different perspectives $([2,3])$. Here we consider the approach adopted in [4]. In this work, timed continuous Petri net (TCPN) models under infinite server semantics are considered. The continuous model thus obtained is piecewise linear with bounded and nonnegative inputs.

\footnotetext{
* This work was partially supported by project CICYT and FEDER DPI2006-15390.
} 
In recent years, a lot of research has been done on controllability of switched linear systems. For instance, [5] and [6] give sufficient and necessary conditions for controllability of 3-dimensional systems and single switching sequence systems, respectively, but always under the assumption of unconstrained inputs. Classic works ([7]) and [8] deal with controllability on linear systems (non piecewise) with bounded and nonnegative inputs, respectively. Camlibel [9] has extended the results of Brammer to a particular class of piecewise linear systems known as linear complementary systems. In [10] optimal control of switched piecewise affine autonomous systems is studied, assuming that the decision variables are the switching instants and the sequence of operating modes.

However, in timed continuous Petri net (TCPN) systems switching is not controllable. Moreover, in [11] it is shown that these systems are not controllable in the classical sense. In [12] it is proven that for Join Free Timed Continuous Petri Nets there exists an invariant set, named Controllability Space (CS), in which the system exhibits the controllability property, i.e. any state of CS is reachable from any other state of CSIn that work, the set CS is characterized.

Here the study of controllability and reachability properties for general timed continuous Petri nets under infinite server semantics is addressed. A controllability notion is presented for TCPN systems. It deals with the possibility that the state evolves from any state, of a given set, to another; so it is an appropriate adaptation of the classical controllability concept of linear continuous systems (see [13]). Based on this controllability definition, the particular structure of TCPN systems allows to obtain a structural characterization of TCPN exhibiting this property. It is worth to remark that this property does not depend on the initial marking, but on the structure and timing of the net as it is proved in the sequel.

This work is organized as follows: in Section 2 an overview of continuous and timed continuous Petri nets is presented, while in Section 3, a concept of controllability is formally introduced. In Section 4, necessary and sufficient conditions for controllability are given, under the hypothesis that all transitions are controllable, while the controllability of systems with uncontrollable transitions is studied in Section 5. Finally, some conclusions are presented in Section 6 .

\section{Basic concepts}

The structure $\mathcal{N}=\langle P, T$, Pre, Post $\rangle$ of continuous Petri nets is the same as the structure of discrete PN. That is, $P$ is a finite set of places, $T$ is a finite set of transitions with $P \cap T=\emptyset$, Pre and Post are $|P| \times|T|$ sized, natural valued, pre- and post-incidence matrices. The main difference is in the evolution rule, since in continuous PN firing is not restricted to be done in integer amounts, and so the marking is not forced to be integer. More precisely, a transition $t$ is enabled at $\mathbf{m}$ iff for every $p \in \bullet^{\bullet} t, \mathbf{m}[p]>0$, and its enabling degree is $\operatorname{enab}(t, \mathbf{m})=\min _{p \in{ }_{t}}\{\mathbf{m}[p] / \operatorname{Pre}[p, t]\}$. The firing of $t$ in a certain amount $\alpha \leq$ $\operatorname{enab}(t, \mathbf{m})$ leads to a new marking $\mathbf{m}^{\prime}=\mathbf{m}+\alpha \cdot \mathbf{C}[P, t]$, where $\mathbf{C}=$ Post - Pre is the token-flow matrix. Right and left rational annullers of $\mathbf{C}$ are called $T$ - 
and $P$-flows, respectively. If there exists $\mathbf{y}>\mathbf{0}(\mathbf{x}>\mathbf{0})$ such that $\mathbf{y} \cdot \mathbf{C}=\mathbf{0}$ $(\mathbf{C} \cdot \mathbf{x}=\mathbf{0})$, the net is said to be conservative (consistent). A set of places $\Sigma$ is a siphon iff $\bullet \subseteq \Sigma^{\bullet}$ (the set of input transitions is contained in the set of output transitions). For reachability, as in [14], the limit concept is used, and a marking reached in the limit of an infinitely long sequence is considered reachable.

For the timing interpretation we will use a first order (or deterministic) approximation of the discrete case ([4]). Hence, a Timed Continuous Petri Net (TCPN) is a continuous PN together with a vector $\boldsymbol{\lambda} \in \mathbb{R}_{>0}^{|T|}$. Here infinite server semantics is considered, thus the flow through a timed transition $t$ is the product of the speed, $\boldsymbol{\lambda}[t]$, and $\operatorname{enab}(t, \mathbf{m})$, the instantaneous enabling, i.e., $\mathbf{f}(\mathbf{m})[t]=\boldsymbol{\lambda}[t] \cdot \operatorname{enab}(t, \mathbf{m})=\boldsymbol{\lambda}[t] \cdot \min _{p \in \bullet_{t}}\{\mathbf{m}[p] / \operatorname{Pre}[p, t]\}$. For the flow to be well defined, every transition must have at least one input place, hence in the following we will assume $\forall t \in T,|\bullet t| \geq 1$. The "min" in the definition leads to the concept of configurations: a configuration assigns to a transition one place that for some markings will control its firing rate (i.e. it is constraining that transition). A good upper bound for the number of configurations is $\prod_{t \in T} \mid \bullet t$.

The flow through the transitions can be written in a vectorial form as $\mathbf{f}(\mathbf{m})=$ $\boldsymbol{\Lambda} \boldsymbol{\Pi}(\mathbf{m}) \mathbf{m}$ (see [11]), where $\boldsymbol{\Lambda}$ is a diagonal matrix whose elements are those of $\boldsymbol{\lambda}$, and $\boldsymbol{\Pi}(\mathbf{m})$ is the configuration operator matrix, defined by elements as

$$
\Pi(\mathbf{m})[i, j]=\left\{\begin{array}{cc}
\frac{1}{\operatorname{Pre}\left[p_{j}, t_{i}\right]} & \text { if } p_{j} \text { is constrainting } t_{i} \\
0 & \text { otherwise }
\end{array}\right.
$$

If more than one place is constrainting the flow of a transition at a given marking, any of them can be used, but only one is taken.

Control action may only be a reduction of the flow through the transitions. Transitions in which a control action can be applied are called controllable. The effective flow through a controllable transition can be represented as: $\mathbf{f}_{i}(\tau)=$ $\boldsymbol{\lambda}\left(t_{i}\right) \cdot \operatorname{enab}(\tau)\left[t_{i}\right]-u(\tau)\left[t_{i}\right]$, where $0 \leq u(\tau)\left[t_{i}\right] \leq \lambda\left(t_{i}\right) \cdot \operatorname{enab}(\tau)\left[t_{i}\right]$.

The control vector $\mathbf{u} \in \mathbb{R}^{|T|}$ is defined such that $\mathbf{u}_{i}$ represents the control action on $t_{i}$. If $t_{i}$ is not controllable then $\mathbf{u}_{i}=0$. The set of all controllable transitions is denoted by $T_{c}$, and the set of uncontrollable transitions is denoted by $T_{n c}$. Then, $T_{c} \cap T_{n c}=\varnothing$ and $T_{c} \cup T_{n c}=T$.

The behavior of a TCPN forced system is described by the state equation:

$$
\begin{gathered}
\stackrel{\bullet}{\mathbf{m}}=\mathbf{C} \Lambda \Pi(\mathbf{m}) \mathbf{m}-\mathbf{C u} \\
\mathbf{0} \leq \mathbf{u} \leq \boldsymbol{\Lambda} \Pi(\mathbf{m}) \mathbf{m}
\end{gathered}
$$

Given a marking trajectory, an input $\mathbf{u}(\mathbf{m})$ (as a function of $\mathbf{m}$ ) such that $\mathbf{0} \leq \mathbf{u}(\mathbf{m}) \leq \mathbf{\Lambda} \boldsymbol{\Pi}(\mathbf{m}) \mathbf{m}$, and $\forall t_{i} \in T_{n c} \mathbf{u}_{i}=0$ along the marking trajectory, is called suitably bounded. Notice that if an input is not suitably bounded for a marking trajectory, then it cannot be applied in this. A marking $\mathbf{m}$ for which $\exists \mathbf{u}$ suitably bounded such that $\mathbf{C}[\mathbf{\Lambda} \Pi(\mathbf{m}) \mathbf{m}-\mathbf{u}]=\mathbf{0}$ is called equilibrium marking.

Marking $\mathbf{m}_{2}$ is said to be reachable from $\mathbf{m}_{1}$ if there exists an input $\mathbf{u}$ that transfers the marking from $\mathbf{m}_{1}$ to $\mathbf{m}_{2}$ in either finite or infinite time (limreachable) and it is suitably bounded. A marking reachable from the initial 
one is simply called reachable. The set of reachable markings can be defined for autonomous continuous PN and TCPN systems [14]. In the sequel, the term reachability always refer to timed systems.

\section{Controllability definition}

Whenever a TCPN system has P-flows, linear dependencies between marking variables appear, introducing state invariants. So, systems with P-flows are not controllable in the classical sense [11]. However, we are interested in the study of controllability "over" this invariant. In the sequel, we refer to this state invariant as $\operatorname{Class}\left(\mathbf{m}_{\mathbf{0}}\right)$, since it is the equivalence class of $\mathbf{m}_{\mathbf{0}}$ under the relation $\beta$ defined as: $\mathbf{m}_{1} \beta \mathbf{m}_{2}$ iff $\mathbf{B}_{\mathbf{y}}{ }^{T} \mathbf{m}_{1}=\mathbf{B}_{\mathbf{y}}{ }^{T} \mathbf{m}_{2}$, where $\mathbf{B}_{\mathbf{y}}$ is a basis of P-flows.

Notice that, for a general TCPN system, every reachable marking belongs to Class $\left(\mathbf{m}_{\mathbf{0}}\right)$. The set Class $\left(\mathbf{m}_{\mathbf{0}}\right)$ can be divided into subsets of markings associated to the same configuration, which are named regions and are denoted by $\Re_{i}=\left\{\mathbf{m} \in \operatorname{Class}\left(\mathbf{m}_{\mathbf{0}}\right) \mid \boldsymbol{\Pi}(\mathbf{m})=\boldsymbol{\Pi}_{i}\right\}$. Notice that such regions are convex sets, and inside each one, the state equation (1) is linear $(\boldsymbol{\Pi}(\mathbf{m})$ is constant). In the sequel, let us denote by $\operatorname{int}\left(\operatorname{Class}\left(\mathbf{m}_{\mathbf{0}}\right)\right)\left(\operatorname{int}\left(\Re_{i}\right)\right)$ the set of interior markings of Class $\left(\mathbf{m}_{\mathbf{0}}\right)\left(\Re_{i}\right)$ considering the space generated by the columns of $\mathbf{C}$.

Next, linear systems controllability definition is recalled [13].

Definition 1. A state equation is fully controllable if there exists an input such that for any two states $\mathbf{x}_{1}$ and $\mathbf{x}_{2}$ of the state space, it is possible to transfer the state from $\mathbf{x}_{1}$ to $\mathbf{x}_{2}$ in finite time.

Notice that this definition cannot be applied to TCPN systems because the set of reachable markings never compose a vector space, as it is inside Class $\left(\mathbf{m}_{\mathbf{0}}\right)$. Moreover, in TCPN systems the input must be suitably bounded (i.e. $\mathbf{0} \leq \mathbf{u} \leq$ $\left.\mathbf{\Lambda} \Pi(\mathbf{m}) \mathbf{m}, \mathbf{u}_{i}=0, \forall t_{i} \in T_{n c}\right)$. Therefore, the following adaptation of the classical controllability definition is proposed.

Definition 2. The TCPN system $\left\langle\mathcal{N}, \boldsymbol{\lambda}, \mathbf{m}_{\mathbf{0}}\right\rangle$ is controllable with bounded input (BIC) over $S \subseteq$ Class $\left(\mathbf{m}_{\mathbf{0}}\right)$ if for any two markings $\mathbf{m}_{1}, \mathbf{m}_{2} \in S$ there exists an input $\mathbf{u}_{12}$ that transfers the system from $\mathbf{m}_{1}$ to $\mathbf{m}_{2}$ in finite or infinite time, and it is suitably bounded (i.e. $\mathbf{0} \leq \mathbf{u}_{12} \leq \mathbf{\Lambda} \Pi(\mathbf{m}) \mathbf{m}$, and $\forall t_{i} \in T_{n c} u_{i}=0$ along the marking trajectory).

Besides, if $S=$ Class $\left(\mathbf{m}_{\mathbf{0}}\right)$ then the system is said to be fully controllable with bounded input (BIFC).

It is important to remark that controllability is a structural property. Even when, under this definition, it is said that a system is controllable over some subset of Class $\left(\mathbf{m}_{\mathbf{0}}\right)$, is the dynamical behavior of the system, which is determined by the structure and timing, that makes the system be controllable or not. 


\section{The case where all transitions are controllable}

In [14] reachability is studied for untimed continuous Petri net systems. An important result introduced in that paper is that a marking $\mathbf{m}$ is reachable iff $\exists \boldsymbol{\sigma} \geq \mathbf{0}$ such that $\mathbf{m}=\mathbf{m}_{\mathbf{0}}+\mathbf{C} \boldsymbol{\sigma} \geq \mathbf{0}$ and the transitions in the support of $\boldsymbol{\sigma}$ are fireable. This result can be extended for TCPN in the following way:

Proposition 1. Let $\left\langle\mathcal{N}, \boldsymbol{\lambda}, \mathbf{m}_{\mathbf{0}}\right\rangle$ be a TCPN system in which $T_{c}=T$. A marking $\mathbf{m}_{1} \in \operatorname{Class}\left(\mathbf{m}_{\mathbf{0}}\right)$ is reachable from $\mathbf{m}_{\mathbf{0}} \in \operatorname{int}\left(\operatorname{Class}\left(\mathbf{m}_{\mathbf{0}}\right)\right)$ iff $\exists \boldsymbol{\sigma} \geq \mathbf{0}$ such that $\mathbf{C} \boldsymbol{\sigma}=\left(\mathbf{m}_{1}-\mathbf{m}_{\mathbf{0}}\right)$.

The following proposition gives a necessary and sufficient condition for controllability over the interior of Class $\left(\mathbf{m}_{\mathbf{0}}\right)$.

Proposition 2. Let $\left\langle\mathcal{N}, \boldsymbol{\lambda}, \mathbf{m}_{\mathbf{0}}\right\rangle$ be a TCPN system, and let $S$ be defined as $S=\left\{\mathbf{m} \in \operatorname{Class}\left(\mathbf{m}_{\mathbf{0}}\right) \mid \mathbf{m}>\mathbf{0}\right\}$. The system $\left\langle\mathcal{N}, \boldsymbol{\lambda}, \mathbf{m}_{\mathbf{0}}\right\rangle$ is $\mathrm{BIC}$ over $S$ iff the net is consistent.

Proof. In [11] a reachability theorem is introduced, according to which, if the net is consistent then the system is BIC over $S$. Now, for the other implication, consider any vector $\mathbf{d} \in \operatorname{span}(\mathbf{C})$ and a marking $\mathbf{m}_{1} \in S$. Then, there exists a scalar $\beta>0$ such that $\mathbf{m}_{1}+\beta \mathbf{d} \geq \mathbf{0}$. Let $\mathbf{m}_{2}=\mathbf{m}_{1}+\beta \mathbf{d}$, then $\mathbf{m}_{2} \in \operatorname{Class}\left(\mathbf{m}_{\mathbf{0}}\right)$. Since the system is $B I C$ over the interior of Class $\left(\mathbf{m}_{\mathbf{0}}\right), \mathbf{m}_{2}$ is a particular solution of the fundamental equation, so $\left(\mathbf{m}_{2}-\mathbf{m}_{1}\right)=\beta \mathbf{d}=\mathbf{C} \boldsymbol{\sigma}$, where $\boldsymbol{\sigma} \geq \mathbf{0}$. Therefore, $\forall \mathbf{d} \in \operatorname{span}(\mathbf{C}), \exists \boldsymbol{\sigma}$ such that $\mathbf{C} \boldsymbol{\sigma}=\mathbf{d}$. Besides, this property implies that $\exists \mathbf{x}>\mathbf{0}$ such that $\mathbf{C x}=\mathbf{0}$, i.e. the net is consistent.

Notice that the condition for controllability (consistency) is purely structural. Actually, the TCPN is BIC over the interior of Class $\left(\mathbf{m}_{\mathbf{0}}\right)$ iff the TCPN is BIC over the interior of Class $\left(\mathbf{m}_{1}\right)$, for every $\mathbf{m}_{1} \geq \mathbf{0}$. Next proposition gives a condition to transfer the marking from the border of Class $\left(\mathbf{m}_{\mathbf{0}}\right)$ to its interior.

Proposition 3. Let $\left\langle\mathcal{N}, \boldsymbol{\lambda}, \mathbf{m}_{\mathbf{0}}\right\rangle$ be a TCPN system. An input $\mathbf{u}$, such that every enabled transition is always fired, transfers the marking from $\mathbf{m}_{\mathbf{0}}$ to some $\mathbf{m}_{f}$, where $\mathbf{m}_{f}$ has not null elements, iff there are not empty siphons at $\mathbf{m}_{\mathbf{0}}$.

Proof. Suppose that $\mathbf{m}_{\mathbf{0}}$ has null elements. Let us define an input $\mathbf{u}$ such that for any enabled transition $t_{j}, \mathbf{u}_{j}<[\boldsymbol{\Lambda} \mathbf{\Pi}(\mathbf{m}) \mathbf{m}]_{j}$. So, a transition $t_{j}$ is such that $[\boldsymbol{\Lambda} \Pi(\mathbf{m}) \mathbf{m}-\mathbf{u}]_{j}=0$ iff there is an input place to $t_{j}$, named $p_{i}$, without tokens. In the same way, $p_{i}$ cannot win tokens iff there exist unmarked input places at all the input transitions of $p_{i}$. So, a place $p_{i}$ has not tokens at time $\tau$ and remains without tokens for future time, iff for every input transition to that place there exists an input place without tokens for all time. Repeating this reasoning to these new input places, it can be seen that $p_{i}$ remains unmarked iff it belongs to an unmarked siphon.

The following theorem introduces necessary and sufficient conditions for controllability over Class $\left(\mathbf{m}_{\mathbf{0}}\right)$. The proof is immediate from previous propositions. 


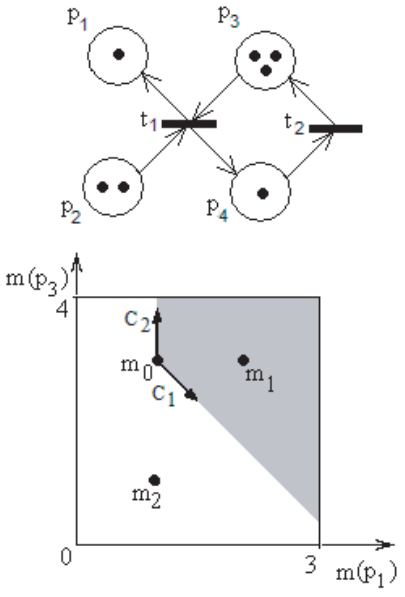

(a)
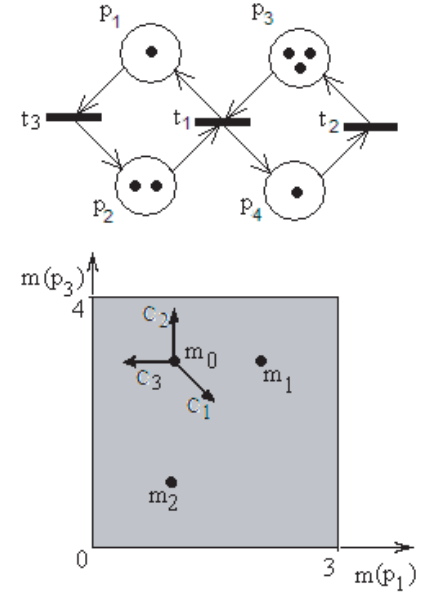

(b)

Fig. 1. (a) TCPN that is not BIFC. (b) A BIFC TCPN system.

Theorem 1. The TCPN system $\left\langle\mathcal{N}, \boldsymbol{\lambda}, \mathbf{m}_{\mathbf{0}}\right\rangle$ is BIFC iff $\mathcal{N}$ is consistent and there do not exist empty siphons at any marking in Class $\left(\mathbf{m}_{\mathbf{0}}\right)$.

Example 1. Consider the TCPN systems of Figure 1, where $\mathbf{m}_{0}=[1,2,3,1]^{T}$, $\mathbf{m}_{1}=[2,1,3,1]^{T}$ and $\mathbf{m}_{2}=[1,2,1,3]^{T}$. Since both systems have 2 P-semiflows, only the marking of two places (in this example, places $p_{1}$ and $p_{3}$ ) are needed to represent the whole state.

Given the system in Figure 1(a), since there exists a vector $\boldsymbol{\sigma} \geq \mathbf{0}$ such that $\mathbf{C} \boldsymbol{\sigma}=\left(\mathbf{m}_{1}-\mathbf{m}_{\mathbf{0}}\right)$, according to Proposition $1, \mathbf{m}_{1}$ is reachable. On the other hand, since $\nexists \boldsymbol{\sigma} \geq \mathbf{0}$ such that $\mathbf{C} \sigma=\left(\mathbf{m}_{2}-\mathbf{m}_{\mathbf{0}}\right)$, then $\mathbf{m}_{2}$ is not reachable. Therefore it is not BIFC. The same conclusion (i.e. the system is not BIFC) can be obtained using Theorem 1. The shadowed area in Figure 1(a) corresponds to the set of reachable markings, notice that it is the convex defined by vectors $\mathbf{C}_{1}$ and $\mathbf{C}_{2}$, which represent the columns of $\mathbf{C}$ restricted to $p_{1}$ and $p_{3}$.

Now, consider the system of Figure 1(b). This system is consistent, so, according to Proposition 2, it is BIC over the interior of Class $\left(\mathbf{m}_{\mathbf{0}}\right)$. Therefore $\mathbf{m}_{1}$ and $\mathbf{m}_{2}$ are reachable from $\mathbf{m}_{\mathbf{0}}$. Besides, since at the border markings of Class $\left(\mathbf{m}_{\mathbf{0}}\right)$ there are not unmarked siphons, according to Theorem 1, the system is BIFC.

\section{Controllability with uncontrollable transitions}

The study of controllability with uncontrollable transitions is more complicated than previous case. In this, consistency is no longer sufficient to guarantee controllability over the interior of Class $\left(\mathbf{m}_{\mathbf{0}}\right)$. Actually, due to the boundedness of the input, a system with uncontrollable transitions is never controllable over 
Class $\left(\mathbf{m}_{\mathbf{0}}\right)$. So, in this case, it is first necessary to define a suitable set of markings, and then, study the controllability over it. In this work only sets of equilibrium markings are considered, because they represent the "stationary operating points" of the modeled system.

The set of all equilibrium markings is defined as:

$E_{q} S=\left\{m \in C l a s s\left(\mathbf{m}_{\mathbf{0}}\right) \mid \exists \mathbf{u}\right.$ suitably bounded such that $\left.\mathbf{C}(\boldsymbol{\Lambda} \Pi(\mathbf{m}) \mathbf{m}-\mathbf{u})=0\right\}$

Notice that if all transitions are controllable then $E_{q} S=\operatorname{Class}\left(\mathbf{m}_{\mathbf{0}}\right)$. The set of all equilibrium markings in the i-th region is defined as $E_{i}=\left\{\mathbf{m} \mid \mathbf{m} \in E_{q} S \cap \Re_{i}\right\}$.

In the sequel, the following notation is adopted. Let $\mathbf{m}^{q} \in E_{q} S$. An equilibrium input for $\mathbf{m}^{q}$ is a vector $\mathbf{u}^{q}$ such that $\mathbf{C}\left(\boldsymbol{\Lambda} \Pi\left(\mathbf{m}^{q}\right) \mathbf{m}^{q}-\mathbf{u}^{q}\right)=\mathbf{0}$ and it is suitably bounded. The equilibrium flow through the transitions for $\mathbf{m}^{q}$ and $\mathbf{u}^{q}$ is denoted as $\mathbf{w}^{q}$, i.e. $\mathbf{w}^{q}=\boldsymbol{\Lambda} \Pi\left(\mathbf{m}^{q}\right) \mathbf{m}^{q}-\mathbf{u}^{q}$.

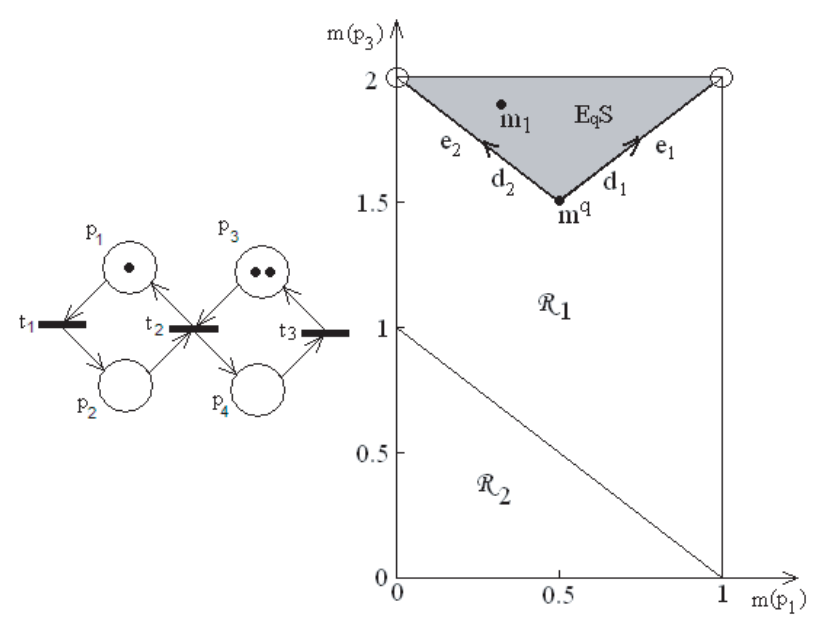

Fig. 2. The set $E_{q} S$, and subsets $E_{i}, E_{i}^{+}$and $E_{i}^{*}$.

Example 2. Consider the system of figure 2 where $\boldsymbol{\Lambda}=\mathbf{I}$ and $T_{c}=\left\{t_{1}, t_{2}\right\}$. There exist two possible configurations: the configuration in which transition $t_{2}$ is constrained by place $p_{2}$, denoted by $\mathcal{C}_{1}$, and the configuration where $t_{2}$ is constrained by $p_{3}$, denoted by $\mathcal{C}_{2} \cdot \Re_{1}$ and $\Re_{2}$ are the regions related to $\mathcal{C}_{1}$ and $\mathcal{C}_{2}$, respectively. The whole triangle with all its edges and vertices corresponds to $E_{1}$. Actually, in this example $E_{1}=E_{q} S$. Since none of the equilibrium markings belongs to the second configuration, then $E_{2}=\varnothing$.

Since the system is linear inside each region, we will first investigate the controllability over each $E_{i}$. For that, it is necessary to represent a given $E_{i}$ in a matrix form. The following definition introduces this representation. 
Definition 3. Let $\left\langle\mathcal{N}, \boldsymbol{\lambda}, \mathbf{m}_{\mathbf{0}}\right\rangle$ be a TCPN system. A Generator of $E_{i} \neq \varnothing$ is a full column rank matrix $\mathbf{G}_{i}$, of order $|P| \times q$ (where $q$ is a suitable natural number), such that:

1. $\forall \mathbf{m}_{1}, \mathbf{m}_{2} \in E_{i}$, the vector $\left(\mathbf{m}_{1}-\mathbf{m}_{2}\right)$ is in the range of $\mathbf{G}_{i}$ (i.e. it is a linear combination of the columns of $\mathbf{G}_{i}$ ).

2. $\mathbf{G}_{i}$ is minimal, i.e. if one of its columns is removed then (1) is no longer true.

Notice that $\mathbf{G}_{i}$ is a kind of basis for $E_{i}$, but, formally speaking, $E_{i}$ is not a vector space, then, it does not have a basis.

Coming back to the system of figure 2, a generator of $E_{1}$ is given by

$$
\mathbf{G}_{1}=\left[\begin{array}{rrrr}
0.5 & -0.5 & 0.5 & 0.5 \\
-0.5 & 0.5 & 0.5 & -0.5
\end{array}\right]^{T}
$$

The restriction of the columns of $\mathbf{G}_{1}$ to the places $p_{1}$ and $p_{3}$ are represented in figure 2 by vectors $\mathbf{d}_{1}$ and $\mathbf{d}_{2}$.

In order to deal with the variable boundedness of the input, controllability is studied through the reachability over neighborhoods of equilibrium markings, because the bounds of the input are almost constant in these. Let us detail this idea. Consider again the system of figure 2 . Let $\mathbf{m}_{1}$ be a marking in the interior $E_{1}$. The evolution of the system, seen from $\mathbf{m}_{1}$, is described by:

$$
\left(\mathbf{m}-\mathbf{m}_{1}\right)=\mathbf{C} \boldsymbol{\Lambda} \Pi\left(\mathbf{m}-\mathbf{m}_{1}\right)-\mathbf{C}\left(\mathbf{u}-\mathbf{u}_{1}\right)
$$

where $\left(\mathbf{u}-\mathbf{u}_{1}\right)$ is the new input. Since $\mathbf{u}_{1}$ is such that $\left[\boldsymbol{\Lambda} \Pi \mathbf{m}_{1}\right]_{j}>\mathbf{u}_{1 j}>0$ for every $t_{j} \in T_{c}\left(\mathbf{m}_{1}\right.$ is in the interior of $\left.E_{1}\right)$, then the entries of $\left(\mathbf{u}-\mathbf{u}_{1}\right)$, related to the controllable transitions, can be settled as either negative or positive values, at least at the markings in a small enough neighborhood of $\mathbf{m}_{1}$. So, the reachability in such neighborhood can be studied through the classical Kalman's reachability condition (see [13]). However, the Kalman's condition cannot be directly applied for all equilibrium markings. Consider the marking $\mathbf{~ m}^{q}$, depicted in figure 2, instead $\mathbf{m}_{1}$. The equilibrium input for this marking is $\mathbf{u}^{q}=\mathbf{0}$, so, the entries of the input $\left(\mathbf{u}-\mathbf{u}^{q}\right)$ can only be settled as nonnegative values (to apply the Kalman's condition it is necessary that the input could take either positive or negative values). Therefore, it is important to know at which markings and at which entries the input can take negative values. For that, the following definitions are introduced.

Definition 4. Let $T_{c}$ be the set of controllable transitions. A transition $t_{j} \in T_{c}$ is said to be fully controllable at $E_{i}$ if there exists an equilibrium marking $\mathbf{m}^{q} \in E_{i}$ with an equilibrium input $\mathbf{u}^{q}$ such that $\left[\boldsymbol{\Lambda} \boldsymbol{\Pi}_{i} \mathbf{m}^{q}\right]_{j}>\mathbf{u}_{j}^{q}>0$. In other case, $t_{j}$ is said to be partially controllable. The set of fully (partially) controllable transitions at $E_{i}$ is denoted as $T_{c f}^{i}\left(T_{c p}^{i}\right)$.

Definition 5. The subset of $E_{i}$, in which the equilibrium flow can be positive, is defined as

$$
E_{i}^{+}=\left\{\mathbf{m}^{q} \in E_{i} \mid \exists \mathbf{u}^{q} \text { such that } \mathbf{w}^{q}>\mathbf{0}\right\}
$$


The subset of $E_{i}$, in which the equilibrium flow can be positive and the entries of the input related to transitions $T_{c f}^{i}$ are positive, is defined as

$$
E_{i}^{*}=\left\{\mathbf{m}^{q} \in E_{i}^{+} \mid \exists \mathbf{u}^{q} \text { such that } \mathbf{u}_{j}^{q}>0, \forall t_{j} \in T_{c f}^{i}\right\}
$$

Notice that $\forall \mathbf{m}^{q} \in E_{i}^{+} \exists \mathbf{u}^{q}$ such that $\mathbf{u}_{j}^{q}<\left[\boldsymbol{\Lambda} \Pi_{i} \mathbf{m}^{q}\right]_{j}, \forall t_{j} \in T_{c}$. For all marking $\mathbf{m}^{q} \in E_{i}^{*}$ the input $\mathbf{u}$, at a neighbor marking $\mathbf{m}$, can be increased or decreased with respect to $\mathbf{u}^{q}$, at those entries related to the transitions in $T_{c f}^{i}$ (i.e. fully controllable transitions). On the other hand, the entries of $\mathbf{u}$ related to the transitions in $T_{c p}^{i}$ can only be increased with respect to $\mathbf{u}^{q}$ (i.e. partially controllable). For instance, the interior of the triangle in figure 2 corresponds to $E_{1}^{*}$, while the union of $E_{1}^{*}$ and the edges $e_{1}$ and $e_{2}$ (without the circled points) corresponds to $E_{1}^{+}$. Besides, $T_{c f}^{1}=T_{c}$ and $T_{c p}^{1}=\varnothing$. Therefore, for any marking in the interior of the triangle (i.e. for any $\mathbf{m}_{1} \in E_{1}^{*}$ ) the input of the system expressed from it (i.e. $\left(\mathbf{u}-\mathbf{u}_{1}\right)$ ), can be settled as either a positive or a negative value at those entries related to the transitions $\left\{t_{1}, t_{2}\right\}$ (i.e. $T_{c f}^{1}$ ).

Remark 1. $E_{i}^{*}, E_{i}^{+}, E_{i}$ are convex sets, and $E_{i}^{*} \subseteq E_{i}^{+} \subseteq E_{i}$. The markings of $\left\{E_{i}^{+}-E_{i}^{*}\right\}$ are limit points, not interiors, of $E_{i}^{*}$ (in the space generated by $\mathbf{G}_{i}$ ).

In the sequel, let us denote as $\mathbf{C}_{c}, \mathbf{C}_{c f}^{i}$ and $\mathbf{C}_{c p}^{i}$ the matrices built with the columns of $\mathbf{C}$ related to the transitions that belong to $T_{c}, T_{c f}^{i}$ and $T_{c p}^{i}$, respectively. In the same way, denote with $\mathbf{u}_{c}, \mathbf{u}_{c f}^{i}$ and $\mathbf{u}_{c p}^{i}$ the vectors built with the entries of $\mathbf{u}$ related to the transitions that belong to $T_{c}, T_{c f}^{i}$ and $T_{c p}^{i}$, respectively. In this way

$$
\mathbf{C u}=\mathbf{C}_{c} \mathbf{u}_{c}=\left[\begin{array}{ll}
\mathbf{C}_{c f}^{i} & \mathbf{C}_{c p}^{i}
\end{array}\right]\left[\begin{array}{l}
\mathbf{u}_{c f}^{i} \\
\mathbf{u}_{c p}^{i}
\end{array}\right]
$$

Now, we are ready to introduce the following theorem, which gives a sufficient and necessary condition for controllability over a set $E_{i}^{+}$.

Theorem 2. Let $\left\langle\mathcal{N}, \boldsymbol{\lambda}, \mathbf{m}_{\mathbf{0}}\right\rangle$ be a TCPN system. Consider $E_{i}^{+}$such that $E_{i}^{+} \cap$ $\operatorname{int}\left(\Re_{i}\right) \neq \varnothing$ and let $\mathbf{G}_{i}$ be a generator of it.

The system is $\mathrm{BIC}$ over $E_{i}^{+}$, considering all marking trajectories in $\Re_{i}$, iff there exist an index $k$ and a matrix $\mathbf{b} \geq \mathbf{0}$ such that

$$
\text { Cont }^{k}\left(\mathbf{C} \boldsymbol{\Lambda} \mathbf{\Pi}_{i},\left[-\mathbf{C}_{c f}^{i} \mathbf{C}_{c f}^{i}-\mathbf{C}_{c p}^{i}\right]\right) \cdot \mathbf{b}=\left[\mathbf{G}_{i}-\mathbf{G}_{i}\right]
$$

where the matrix function $\operatorname{Cont}^{k}(\mathbf{A}, \mathbf{B})=\left[\begin{array}{lllll}\mathbf{B} & \mathbf{A B} & \ldots & \mathbf{A}^{k} \mathbf{B}\end{array}\right]$.

Proof. The proof is large and to improve readability it is shown in the appendix.

This theorem also includes the result introduced in previous section, in which consistency is sufficient to guarantee controllability over $\operatorname{int}\left(\operatorname{Class}\left(\mathbf{m}_{\mathbf{0}}\right)\right)$, where $T_{c}=T$. This is easy to see noting that matrix $\left[-\mathbf{C}_{c f}^{i} \mathbf{C}_{c f}^{i}-\mathbf{C}_{c p}^{i}\right]$ includes all columns of the incidende matrix, and since the net is consistent, there always exists $\mathbf{b} \geq 0$ that fulfills the condition (consider $k=0$ ). 
The condition of previous theorem could be difficult to check, since there is no bound for the index $k$. Next corollary separates this condition into a necessary and a sufficient conditions that can be checked in polynomial time.

Corollary 1. Let $\left\langle\mathcal{N}, \boldsymbol{\lambda}, \mathbf{m}_{\mathbf{0}}\right\rangle$ be a TCPN system. Consider some $E_{i}^{+}$such that $E_{i}^{+} \cap \operatorname{int}\left(\Re_{i}\right) \neq \varnothing$, as previously defined, and let $\mathbf{G}_{i}$ be a generator of it. Then:

1. If $\exists \mathbf{b}$ such that $C$ ont ${ }^{|P|-1}\left(\mathbf{C} \mathbf{\Lambda} \mathbf{\Pi}_{i},-\mathbf{C}_{c f}^{i}\right) \cdot \mathbf{b}=\mathbf{G}_{i}$, then the system is controllable over $E_{i}^{+}$. Besides, if $T_{c f}^{i}=T_{c}$ then it is also a necessary condition for controllability over $E_{i}^{+}$, considering all the marking trajectories in $\Re_{i}$.

2. If $\nexists \mathbf{b}$ such that Cont ${ }^{|P|-1}\left(\mathbf{C} \boldsymbol{\Lambda} \boldsymbol{\Pi}_{i},-\mathbf{C}_{c}\right) \cdot \mathbf{b}=\mathbf{G}_{i}$, then the system is not controllable over $E_{i}^{+}$, considering all marking trajectories in $\Re_{i}$.

Proof. Statement 1). Suppose that $\exists \mathbf{b}$ such that $\operatorname{Cont}^{|P|-1}\left(\mathbf{C} \boldsymbol{\Lambda} \boldsymbol{\Pi}_{i},-\mathbf{C}_{c f}^{i}\right) \cdot \mathbf{b}=$ $\mathbf{G}_{i}$. Then, $\exists \mathbf{b}^{\prime} \geq \mathbf{0}$ such that $C o n t^{|P|-1}\left(\mathbf{C} \boldsymbol{\Lambda} \boldsymbol{\Pi}_{i},\left[-\mathbf{C}_{c f}^{i}, \mathbf{C}_{c f}^{i}\right]\right) \cdot \mathbf{b}^{\prime}=\left[\mathbf{G}_{i},-\mathbf{G}_{i}\right]$. So, according to Theorem 2 , the system is BIC over $E_{i}^{+}$. On the other hand, suppose that $T_{c}=T_{c f}^{i}$, so $\mathbf{C}_{c f}=\mathbf{C}_{c}$. If $\mathbf{G}_{i}$ is not in the range of $C o n t^{|P|-1}\left(\mathbf{C} \boldsymbol{\Lambda} \mathbf{\Pi}_{i},-\mathbf{C}_{c f}^{i}\right)$ then it is not in the range of $C o n t^{|P|-1}\left(\mathbf{C} \boldsymbol{\Lambda} \mathbf{\Pi}_{i},-\mathbf{C}_{c}^{i}\right)$. This condition is equal to that of statement 2).

Statement 2). Suppose that $\nexists \mathbf{b}$ such that Cont $^{|P|-1}\left(\mathbf{C} \boldsymbol{\Lambda} \mathbf{\Pi}_{i},-\mathbf{C}_{c}\right) \cdot \mathbf{b}=\mathbf{G}_{i}$, then $\mathbf{G}_{i}$ is not in the range of $\operatorname{Cont}^{k}\left(\mathbf{C} \boldsymbol{\Lambda} \mathbf{\Pi}_{i},\left[-\mathbf{C}_{c f}^{i}, \mathbf{C}_{c f}^{i},-\mathbf{C}_{c p}^{i}\right]\right)$, for $k=|P|-1$, and according to the Calley-Hamilton's theorem, $\mathbf{G}_{i}$ is not in the range for any index $k$, so, the system is not BIC over $E_{i}^{+}$(Theorem 2).

Notice that previous corollary does not consider all possible cases, for that, next proposition introduces an equivalent condition to that of Theorem 2. The proof of this proposition is presented in the appendix.

Proposition 4. Suppose that all the coefficients of the characteristic polynomial of a matrix $\mathbf{A}$ are nonnegative.

There exist $k$ and $\mathbf{X}_{k} \leq \mathbf{0}$ such that $\operatorname{Cont}^{k}(\mathbf{A}, \mathbf{B}) \cdot \mathbf{X}_{k}=\mathbf{Y}$ iff $\exists \mathbf{X}_{2 n-1} \leq \mathbf{0}$ such that Cont $^{2 n-1}(\mathbf{A}, \mathbf{B}) \cdot \mathbf{X}_{2 n-1}=\mathbf{Y}$, where $n$ is the order of $\mathbf{A}$.

Example 3. Consider the system of figure 3 with $T_{c}=\left\{t_{4}\right\}, \lambda_{1}=\lambda_{2}=\lambda_{3}=1$ and $\lambda_{4}=2$. Four configurations are realizable in the net, those are characterized by: $\mathcal{C}_{1}=\left\{\left(t_{2}, p_{2}\right),\left(t_{3}, p_{4}\right)\right\}, \mathcal{C}_{2}=\left\{\left(t_{2}, p_{3}\right),\left(t_{3}, p_{4}\right)\right\}, \mathcal{C}_{3}=\left\{\left(t_{2}, p_{2}\right),\left(t_{3}, p_{5}\right)\right\}$ and $\mathcal{C}_{4}=\left\{\left(t_{2}, p_{3}\right),\left(t_{3}, p_{5}\right)\right\}$, (the arcs $\left(t_{1}, p_{1}\right)$ and $\left(t_{4}, p_{6}\right)$ are also present in all the configurations). Moreover, given the initial marking of this system, $\mathcal{C}_{2}$ cannot occur $\left(p_{3}\right.$ and $p_{4}$ cannot constraint its respective output transitions at the same time). The lines inside the polytope correspond to set $E_{q} S$. The markings in the segment $\left[\mathbf{m}_{1}, \mathbf{m}_{2}\right]$ correspond to $E_{1}$, the markings in $\left[\mathbf{m}_{2}, \mathbf{m}_{3}\right]$ correspond to $E_{3}$ and the markings in $\left[\mathbf{m}_{3}, \mathbf{m}_{4}\right]$ correspond to $E_{4}$. For this system we have that $T_{c f}^{1}=T_{c f}^{3}=T_{c f}^{4}=\left\{t_{4}\right\}$, and

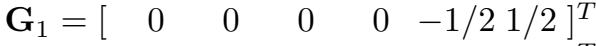

$$
\begin{aligned}
& \mathbf{G}_{3}=\left[\begin{array}{ccccccc}
0 & 0 & -1 & 1 & 0 & 0
\end{array}\right]^{T} \\
& \mathbf{G}_{4}=[-1 / 31 / 3-1 / 31 / 3-1 / 31 / 3]^{T}
\end{aligned}
$$




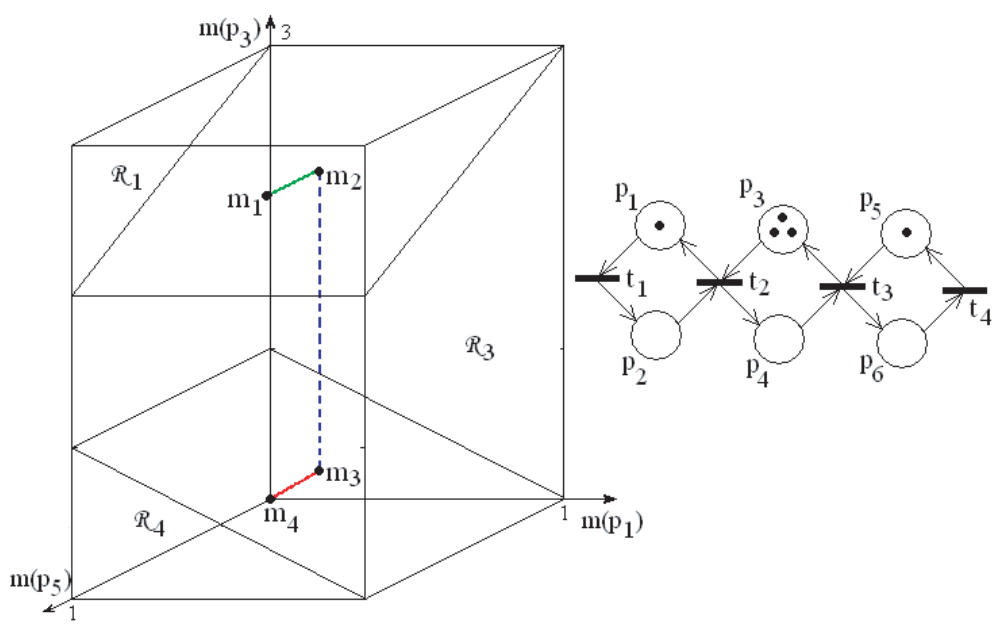

Fig. 3. TCPN system with its $E_{q} S$. Transition $t_{4}$ is the only controllable one

Since $T_{c f}^{i}=T_{c}$ for the three configurations, we can check for the condition of Corollary 1 to investigate the controllability over each $E_{i}^{+}$, i.e. for every $E_{i}^{+}$ investigate if $\mathbf{G}_{i}$ is in the range of $\operatorname{Cont}^{5}\left(\mathbf{C} \boldsymbol{\Lambda} \boldsymbol{\Pi}_{i},-\mathbf{C}_{c f}^{i}\right)$. In this case the system fulfills that condition for the three $E_{i}^{+}$, so, it is BIC over each one.

Now, consider the same system but with $\boldsymbol{\Lambda}=\mathbf{I}$. In this case, the sets $E_{3}$ and $E_{4}$ remain unchanged, and so $\mathbf{G}_{3}$ and $\mathbf{G}_{4}$, but $E_{1}=\left\{\mathbf{m}_{2}\right\}$. Also, $T_{c f}^{3}=\varnothing$ and $T_{c f}^{4}=t_{4}$. Checking for the condition of Corollary 1 (a), it can be concluded that the system is BIC over $E_{4}$. However, since $T_{c f}^{3}=\varnothing$, we cannot apply the same corollary for $E_{3}$ (the system does not fulfill the condition of neither statement a) nor b)). In such case, since the coefficients of the characteristic polynomial of $\mathbf{C} \boldsymbol{\Lambda} \Pi_{3}$ are nonnegative, we can use Proposition 4 to investigate if the system fulfills the condition of Theorem 2 (i.e. the system is BIC over $E_{3}^{+}$iff $\exists \mathbf{b} \geq \mathbf{0}$ such that $\left.\operatorname{Cont}^{2|P|-1}\left(\mathbf{C} \boldsymbol{\Lambda} \mathbf{\Pi}_{3},-\mathbf{C}_{c p}^{3}\right) \cdot \mathbf{b}=\left[\mathbf{G}_{3},-\mathbf{G}_{3}\right]\right)$. Since there does not exist a nonnegative solution $\mathbf{b}$, we can concluded that the system is not BIC over $E_{3}^{+}$.

Finally, next proposition introduces sufficient conditions for controllability over the union of sets of equilibrium markings of different regions.

Proposition 5. Let $\left\langle\mathcal{N}, \boldsymbol{\lambda}, \mathbf{m}_{\mathbf{0}}\right\rangle$ be a TCPN system. Consider some equilibrium sets $E_{1}^{+}, E_{2}^{+}, \ldots, E_{j}^{+}$as defined above. If the system is BIC over each one and their union (i.e. $\bigcup_{i=1}^{j} E_{i}^{+}$) is connected, then the system is $\mathrm{BIC}$ over the union.

Proof. Consider two of those sets $E_{1}^{+}, E_{2}^{+}$such that $E_{1}^{+} \cap E_{2}^{+} \neq \varnothing$. Let $\mathbf{m}^{q}$ be a marking such that $\mathbf{m}^{q} \in E_{1}^{+} \cap E_{2}^{+}$. Since the system is controllable over $E_{1}^{+}$and $E_{2}^{+}$, there exists a marking $\mathbf{m}_{2} \in E_{2}^{+}-E_{1}^{+}$that is reachable, in finite time, from another marking in $\mathbf{m}_{1} \in E_{1}^{+}-E_{2}^{+}$, due to the fact that both are reachable from $\mathbf{m}^{q}$, in finite time, and to the continuity of the flow function. Then, any marking 
of $E_{2}^{+}$is reachable from any marking of $E_{1}^{+}$, via $\mathbf{m}_{1}$ and $\mathbf{m}_{2}$. Following a similar reasoning, it can be concluded that the system is controllable over $\bigcup_{i=1}^{j} E_{i}^{+}$.

Example 4. Consider the system of figure 3 , where $T_{c}=\left\{t_{4}\right\}, \lambda_{1}=\lambda_{2}=\lambda_{3}=1$ and $\lambda_{4}=2$. In the previous example it was shown that the system is controllable over each $E_{i}^{+}$. Now, since the union of $E_{1}^{+}, E_{3}^{+}$and $E_{4}^{+}$is connected, then, according to Proposition 5, the system is BIC over $E_{1}^{+} \cup E_{3}^{+} \cup E_{4}^{+}$. Notice that, the union of those sets is equal to $E_{q} S-\left\{\mathbf{m}_{4}\right\}$, i.e. the set of all the equilibrium markings inside the interior of Class $\left(\mathbf{m}_{\mathbf{0}}\right)$.

\section{Conclusions}

This work addresses the controllability of timed continuous Petri Nets $(T C P N)$ from a structural point of view. The main contributions of this work are focused in defining the controllability property and its characterization. The definition of controllability for TCPN systems is introduced as an adaptation of that defined for linear continuous systems. For the case where all transitions are controllable, a polynomial characterization of controllable TCPN systems is presented. For systems with uncontrollable transitions, sufficient and necessary conditions for controllability, over subsets of equilibrium markings, are introduced, and sufficient conditions for controllability, over the union of those subsets, are given.

\section{References}

1. Mahulea, C., Recalde, L., Silva, M.: On performance monotonicity and basic servers semantics of continuous Petri nets. In: 8th Int. Workshop on Discrete Event Systems WODES06, Ann Arbor, USA, IEEE Computer Society Press (2006) 345-351

2. Alla, H., David, R.: Continuous and hybrid Petri nets. Journal of Circuits, Systems, and Computers 8(1) (1998) 159-188

3. Recalde, L., Teruel, E., Silva, M.: Autonomous continuous P/T systems. In Donatelli, S., Kleijn, J., eds.: Application and Theory of Petri Nets 1999. Volume 1639 of LNCS., Springer (1999) 107-126

4. Silva, M., Recalde, L.: Petri nets and integrality relaxations: A view of continuous Petri nets. IEEE Trans. on Systems, Man, and Cybernetics 32(4) (2002) 314-327

5. Sun, Z., Zheng, D.: On stabilization of switched linear control systems. IEEE Trans. on Automatic Control 46(2) (2001) 291-295

6. Xie, G., Wang, L.: Controllability and stabilization of switched linear systems. Systems and Control Letters 48(2) (2002) 135-155

7. Sontag, E.: An algebraic approach to bounded controllability of linear systems. Int. Journal of Control 39 (1984) 181-188

8. Brammer, R.: Controllability in linear autonomous systems with positive controllers. SIAM J. Control 10(2) (1972) 329-353

9. Camlibel, M.: Popov-belevitch-hautus type controllability for linear complementary systems. IEEE Trans. on Software Engineering 56(5) (2007) 381-387

10. Seatzu, C., Corona, D., Giua, A., Bemporad, A.: Optimal control of continuoustime switched affine systems. IEEE Trans. on Automatic Control 51(5) (2006) $726-741$ 
11. Mahulea, C., Ramirez-Trevino, A., Recalde, L., Silva, M.: Steady state control reference and token conservation laws in continuous Petri net systems. IEEE Trans. on Automation Science and Engineering (2007) Aceptado para su publicacin.

12. Jiménez, E., Júlvez, J., Recalde, L., Silva, M.: On controllability of timed continuous Petri net systems: the join free case. In: Proc. of the 44th IEEE Conf. on Decision and Control (Joint CDC-ECC), Seville, Spain (2005) 7645-7650

13. Chen, C.: Linear system theory and design. Oxford University Press USA (1984)

14. Júlvez, J., Recalde, L., Silva, M.: On reachability in autonomous continuous Petri net systems. In van der Aalst, W., Best, E., eds.: Proc. of the $24^{\text {th }}$ Int. Conf. on Application and Theory of Petri Nets (ICATPN 2003). Volume 2679. Springer, Eindhoven, The Netherlands (June 2003) 221-240

\section{Additional proofs}

Proof (of Theorem 2). This proof is presented as follows: first, the state equation is rewritten, next, considering the solution of this new equation, it is proved that all equilibrium markings in a neighborhood of any marking of $E_{i}^{*} \cap \operatorname{int}\left(\Re_{i}\right)$ are reachable iff the condition is fulfilled, and so, since this set can be covered by these reachable neighborhoods then the system is BIC over $E_{i}^{*} \cap \operatorname{int}\left(\Re_{i}\right)$ iff the condition is fulfilled. Finally, it is proven that the system is controllable over $E_{i}^{*} \cap \operatorname{int}\left(\Re_{i}\right)$ iff it is controllable over $E_{i}^{+}$.

If $E_{i}^{*}=\varnothing$ then consider the set $E_{i}^{+}$instead of $E_{i}^{*}$.

Consider an equilibrium marking $\left(\mathbf{m}^{q}, \mathbf{u}^{q}\right)$, where $\mathbf{m}^{q} \in E_{i}^{*} \cap \operatorname{int}\left(\Re_{i}\right)$. The state equation for any marking in $\Re_{i}$ can be expressed as:

$$
\stackrel{\bullet}{\mathbf{m}}=\left(\mathbf{m}-\dot{\mathbf{m}}^{q}\right)=\mathbf{C} \Lambda \Pi_{i}\left(\mathbf{m}-\mathbf{m}^{q}\right)-\mathbf{C}\left(\mathbf{u}-\mathbf{u}^{q}\right)
$$

The solution of this state equation is given by:

$$
\mathbf{m}(\tau)-\mathbf{m}^{q}=e^{\mathbf{C} \boldsymbol{\Lambda} \boldsymbol{\Pi}_{i} \tau}\left(\mathbf{m}_{\mathbf{0}}-\mathbf{m}^{q}\right)-\int_{0}^{\tau} e^{\mathbf{C} \boldsymbol{\Lambda} \boldsymbol{\Pi}_{i} \zeta} \mathbf{C}\left(\mathbf{u}(\tau-\zeta)-\mathbf{u}^{q}\right) d \zeta
$$

Now, let us analyze the boundedness of the input. By definition of $T_{c f}^{i}, T_{c p}^{i}$ and $\mathbf{m}^{q}$, the input $\mathbf{u}^{q}$ is such that $\left[\boldsymbol{\Lambda} \boldsymbol{\Pi}_{i} \mathbf{m}^{q}\right]_{j}>\mathbf{u}_{j}^{q}>\mathbf{0} \forall t_{j} \in T_{c f}^{i}, \mathbf{u}_{j}^{q}=0 \forall t_{j} \in$ $T_{c p}^{i}$, and $\mathbf{u}_{j}=0 \forall t_{j} \in T_{n c}$. Then, at any marking of a small enough neighborhood of $\mathbf{m}^{q}$ the value of $\left(\mathbf{u}(\tau-\zeta)-\mathbf{u}^{q}\right)_{j}$ can be settled as either positive or negative $\forall t_{j} \in T_{c f}^{i}$, as nonnegative $\forall t_{j} \in T_{c p}^{i}$, and zero $\forall t_{j} \in T_{n c}$. Besides, since $\mathbf{m}^{q} \in E_{i}^{*} \cap$ $\operatorname{int}\left(\Re_{i}\right)$, it can be demonstrated that $\mathbf{m}^{q}$ is an interior point of this neighborhood, considering the space generated by the columns of $\operatorname{Cont}^{k}\left(\mathbf{C} \boldsymbol{\Lambda} \boldsymbol{\Pi}_{i}, \mathbf{C}_{c}\right)$. Therefore, considering the notation previously introduced, we can define column vectors $\mathbf{u}^{+}$ and $\mathbf{u}^{-}$such that $\mathbf{C}_{c f}^{i}\left(\mathbf{u}_{c f}^{i}-\mathbf{u}_{c f}^{q i}\right)=\left[\mathbf{C}_{c f}^{i}-\mathbf{C}_{c f}^{i}\right] \cdot\left[\mathbf{u}^{+} \mathbf{u}^{-}\right]^{T}$ where $\mathbf{u}^{+}, \mathbf{u}^{-} \geq \mathbf{0}$.

Then $\mathbf{C u}=\left[\mathbf{C}_{c f}^{i}-\mathbf{C}_{c f}^{i} \mathbf{C}_{c p}^{i}\right] \mathbf{u}^{*}$ where $\mathbf{u}^{*}=\left[\mathbf{u}^{+} \mathbf{u}^{-}\left(\mathbf{u}_{c p}^{i}-\mathbf{u}_{c p}^{q i}\right)\right]^{T} \geq 0$ at any marking in a neighborhood of $\mathbf{m}^{q}$.

Considering $\mathbf{m}_{\mathbf{0}}=\mathbf{m}^{q}$ and substituting previous equation into (2), we obtain:

$$
\mathbf{m}(\tau)-\mathbf{m}^{q}=-\int_{0}^{\tau} e^{\mathbf{C} \Lambda \Pi_{i} \zeta}\left[\mathbf{C}_{c f}^{i}-\mathbf{C}_{c f}^{i} \mathbf{C}_{c p}^{i}\right] \mathbf{u}^{*}(\tau-\zeta) d \zeta
$$


Expanding the exponential matrix and taking the constant elements out of the integral, we obtain

$$
\begin{aligned}
& \mathbf{m}(\tau)-\mathbf{m}^{q}=\left[\mathbf{I}\left(\mathbf{C} \boldsymbol{\Lambda} \boldsymbol{\Pi}_{i}\right)\left(\mathbf{C} \boldsymbol{\Lambda} \boldsymbol{\Pi}_{i}\right)^{2} \ldots\right] . \\
& {\left[-\mathbf{C}_{c f}^{i} \mathbf{C}_{c f}^{i}-\mathbf{C}_{c p}^{i}\right]\left[\begin{array}{c}
\int_{0}^{\tau} \mathbf{u}^{*}(\tau-\zeta) d \zeta \\
\int_{0}^{\tau} \zeta \mathbf{u}^{*}(\tau-\zeta) d \zeta \\
\int_{0}^{\tau} \frac{\zeta^{2}}{2} \mathbf{u}^{*}(\tau-\zeta) d \zeta \\
:
\end{array}\right]}
\end{aligned}
$$

Notice that the entries of the right side vector are linearly independent and nonnegative functions, for a small enough neighborhood of $\mathbf{m}^{q}$. Moreover, previous equation constitutes a necessary and sufficient condition for reachability.

If the condition of this theorem is fulfilled then, by definition of $\mathbf{G}_{i}$, for any equilibrium marking $\mathbf{m}^{r}$ in $\Re_{i}$ there exists $\mathbf{b}^{r} \geq \mathbf{0}$ such that

$$
\mathbf{m}^{r}-\mathbf{m}^{q}=\left[\mathbf{I}\left(\mathbf{C} \boldsymbol{\Lambda} \boldsymbol{\Pi}_{i}\right)\left(\mathbf{C} \boldsymbol{\Lambda} \boldsymbol{\Pi}_{i}\right)^{2} \ldots\right] \cdot\left[-\mathbf{C}_{c f}^{i} \mathbf{C}_{c f}^{i}-\mathbf{C}_{c p}^{i}\right] \mathbf{b}^{r}
$$

The entries of $\mathbf{b}^{r} \geq \mathbf{0}$ could be as small as desired (just considering $\mathbf{m}^{r}$ close enough to $\mathbf{m}^{q}$ ), so, comparing this equation with (3), it can be concluded that $\mathbf{m}^{r}$ is reachable from $\mathbf{m}^{q}$. Then, there exists a neighborhood of $\mathbf{m}^{q}$ in which all equilibrium markings are reachable from $\mathbf{m}^{q}$. Finally, since $E_{i}^{*} \cap \operatorname{int}\left(\Re_{i}\right)$ is a convex set and $\forall \mathbf{m}^{q} \in E_{i}^{*} \cap \operatorname{int}\left(\Re_{i}\right)$ there exists such reachable neighborhood, in which $\mathbf{m}^{q}$ is an interior point, then the set $E_{i}^{*} \cap \operatorname{int}\left(\Re_{i}\right)$ is covered by these reachable neighborhoods and the system is BIC over $E_{i}^{*} \cap \operatorname{int}\left(\Re_{i}\right)$.

On the other hand, notice that at any marking in $\Re_{i}$ the input is such that $\mathbf{u}^{*} \geq \mathbf{0}$. So, if the condition asked for the theorem is not fulfilled, then $\exists \mathbf{m}^{r}$ for which $\nexists \mathbf{b}^{r} \geq \mathbf{0}$ that fulfills previous equation, and according to equation (3), there does not exist a marking trajectory from $\mathbf{m}^{q}$ to $\mathbf{m}^{r}$ in $\Re_{i}$ in which $\mathbf{u}^{*} \geq \mathbf{0}$, therefore, $\mathbf{m}^{r}$ is not reachable from $\mathbf{m}^{q}$, considering all the marking trajectories in $\Re_{i}$ and so, the system is not BIC over $E_{i}^{*}$.

Now, let us demonstrate that controllability over $E_{i}^{*} \cap \operatorname{int}\left(\Re_{i}\right)$ implies controllability over $E_{i}^{+}$. For this, suppose that the system is controllable over $E_{i}^{*} \cap$ $\operatorname{int}\left(\Re_{i}\right)$. Consider any marking $\mathbf{m}^{r} \in E_{i}^{+}-\left(E_{i}^{*} \cap \operatorname{int}\left(\Re_{i}\right)\right)$ and the markings $\mathbf{m}^{q}, \mathbf{m}^{r \prime}, \mathbf{m}^{q \prime} \in E_{i}^{*} \cap \operatorname{int}\left(\Re_{i}\right)$, such that $\left(\mathbf{m}^{r}-\mathbf{m}^{q}\right)=\alpha\left(\mathbf{m}^{r \prime}-\mathbf{m}^{q \prime}\right)$ with $\alpha \in \mathbb{R}_{>0}$.

Since the system is BIC over $E_{i}^{*} \cap \operatorname{int}\left(\Re_{i}\right)$, then $\exists \mathbf{u}^{\prime}$ that transfers the state from $\mathbf{m}^{r \prime}$ to $\mathbf{m}^{q \prime}$. So, by linearly, an input $\mathbf{u}$ such that $\left(\mathbf{u}-\mathbf{u}^{q}\right)=\alpha\left(\mathbf{u}^{\prime}-\mathbf{u}^{q \prime}\right)$ transfers the state from $\mathbf{m}^{r}$ to $\mathbf{m}^{q}$ (this is easy to prove by using equation (2)). Actually, $\left(\mathbf{m}(\tau)-\mathbf{m}^{q}\right)=\alpha\left(\mathbf{m}^{\prime}(\tau)-\mathbf{m}^{q \prime}\right)$, where $\mathbf{m}(\tau)\left(\mathbf{m}^{\prime}(\tau)\right)$ is the marking at time $\tau$ if $\mathbf{m}_{\mathbf{0}}=\mathbf{m}^{q}\left(\mathbf{m}_{\mathbf{0}}=\mathbf{m}^{q \prime}\right)$ and $\mathbf{u}\left(\mathbf{u}^{\prime}\right)$ is applied. So, choosing a suitable trajectory for $\mathbf{m}^{\prime}(\tau)$ in $E_{i}^{*} \cap \operatorname{int}\left(\Re_{i}\right)$, we can make $\mathbf{m}(\tau)$ stay always inside $\Re_{i}$. Then, $\mathbf{m}^{q}$ is reachable from $\mathbf{m}^{r}$ if $\mathbf{u}$ is suitably bounded.

Analyzing the boundedness of the input, since $\mathbf{0} \leq \mathbf{u}^{\prime} \leq \boldsymbol{\Lambda} \boldsymbol{\Pi}_{i} \mathbf{m}^{\prime}$ then $-\alpha \mathbf{u}^{q \prime} \leq \alpha\left(\mathbf{u}^{\prime}-\mathbf{u}^{q \prime}\right) \leq \alpha\left(\boldsymbol{\Lambda} \boldsymbol{\Pi}_{i} \mathbf{m}^{\prime}-\mathbf{u}^{q^{\prime}}\right)$. Now, substituting $\mathbf{u}^{\prime}$ and $\mathbf{m}^{\prime}$ and arranging the terms, we obtain: $\left(\mathbf{u}^{q}-\alpha \mathbf{u}^{q \prime}\right) \leq \mathbf{u} \leq \boldsymbol{\Lambda} \boldsymbol{\Pi}_{i} \mathbf{m}-\left(\mathbf{w}^{q}-\alpha \mathbf{w}^{q \prime}\right)$. Since $\mathbf{w}^{q}>\mathbf{0}$ and $\left(\mathbf{u}_{j}^{q \prime}>0 \Rightarrow \mathbf{u}_{j}^{q}>0\right)$, then $\exists \alpha>0$ small enough such that $\mathbf{u}^{q} \geq \alpha \mathbf{u}^{q \prime}$ 
and $\mathbf{w}^{q} \geq \alpha \mathbf{w}^{q \prime}$, so, $\mathbf{0} \leq \mathbf{u} \leq \boldsymbol{\Lambda} \boldsymbol{\Pi}_{i} \mathbf{m}$, i.e. $\mathbf{u}$ is suitably bounded (by definition of $\left.\mathbf{u}, \forall t_{j} \in T_{n c} \mathbf{u}_{j}=0\right)$.

Therefore, for any marking $\mathbf{m}^{r} \in E_{i}^{+}-\left(E_{i}^{*} \cap \operatorname{int}\left(\Re_{i}\right)\right)$ there exists a marking $\mathbf{m}^{q} \in E_{i}^{*} \cap \operatorname{int}\left(\Re_{i}\right)$ reachable from $\mathbf{m}^{r}$. Besides, notice that the points of $E_{i}^{+}-$ $\left(E_{i}^{*} \cap \operatorname{int}\left(\Re_{i}\right)\right)$ are limit points of $E_{i}^{+} \cap \operatorname{int}\left(\Re_{i}\right)$, so, every marking in $E_{i}^{+}-$ $\left(E_{i}^{*} \cap \operatorname{int}\left(\Re_{i}\right)\right)$ can be reached (at least in infinite time) from any marking in $E_{i}^{*} \cap \operatorname{int}\left(\Re_{i}\right)$, following a trajectory in $E_{i}^{*} \cap \operatorname{int}\left(\Re_{i}\right)$. So, the system is controllable over $E_{i}^{+}$if it is controllable over $E_{i}^{*} \cap \operatorname{int}\left(\Re_{i}\right)$.

Finally, by definition, if the system is not controllable over $E_{i}^{*} \cap \operatorname{int}\left(\Re_{i}\right)$, considering all marking trajectories in $\Re_{i}$, then it is not BIC over $E_{i}^{+}$.

Proof (of Proposition 4). Let $m$ be the number of columns of $\mathbf{B}$.

(Sufficiency) Suppose that there exists $\mathbf{X}_{2 n-1} \leq \mathbf{0}$ such that $C_{\text {ont }}{ }^{2 n-1}(\mathbf{A}, \mathbf{B})$. $\mathbf{X}_{2 n-1}=\mathbf{Y}$. So, consider $k$ as $2 n-1$.

(Necessity) First, let us introduce some useful equalities.

According to the Calley-Hamilton's theorem $\mathbf{I} \alpha_{0}+\mathbf{A} \alpha_{1}+\ldots+\mathbf{A}^{n} \alpha_{n}=\mathbf{0}$ where $\left\{\alpha_{0}, \alpha_{1}, \ldots, \alpha_{n}\right\}$ are the coefficients of the characteristic polynomial of A. Without lost of generality, suppose that $\alpha_{n}=1$. Now, post-multiplying this equation by $\mathbf{B}$ and arranging the terms, we obtain

$$
\left[\begin{array}{llll}
\mathbf{B} & \mathbf{A B} & \cdots & \mathbf{A}^{n-1} \mathbf{B}
\end{array}\right]\left[\begin{array}{c}
\mathbf{I} \alpha_{0} \\
\mathbf{I} \alpha_{1} \\
\vdots \\
\mathbf{I} \alpha_{n-1}
\end{array}\right]=-\mathbf{A}^{n} \mathbf{B}
$$

where $\mathbf{I}$ is the unity matrix of order $m$ (the number of columns of $\mathbf{B}$ ).

Define $\hat{\mathbf{a}}=\left[\begin{array}{llll}\mathbf{I} \alpha_{0} & \mathbf{I} \alpha_{1} & \ldots & \mathbf{I} \alpha_{n-1}\end{array}\right]^{T}$, and let $\mathbf{0}_{k m \times m}$ be a null matrix of order $k m \times m$. So, according to the previous equation

$$
-\mathbf{A}^{n+k} \mathbf{B}=\mathbf{A}^{k}\left[\mathbf{B} \mathbf{A B} \ldots \mathbf{A}^{n-1} \mathbf{B}\right] \hat{\mathbf{a}}=\operatorname{Cont}^{n+k-1}\left[\begin{array}{c}
\mathbf{0}_{k m \times m} \\
\hat{\mathbf{a}}
\end{array}\right]
$$

Now, every solution $\mathbf{X}_{k}$ for

$$
\operatorname{Cont}^{k}(\mathbf{A}, \mathbf{B}) \cdot \mathbf{X}_{k}=\mathbf{Y}
$$

has the form: $\mathbf{X}_{k}=\mathbf{X}_{k}^{p}+\operatorname{ker}\left\{C o n t^{k}(\mathbf{A}, \mathbf{B})\right\} \gamma$, where $\mathbf{X}_{k}^{p}$ is a particular solution and $\operatorname{ker}\left\{\operatorname{Cont}^{k}(\mathbf{A}, \mathbf{B})\right\} \gamma$ is an element in the kernel of $\operatorname{Cont}^{k}(\mathbf{A}, \mathbf{B})$. In this way, it is easy to prove, using equation (4), that any solution $\mathbf{X}_{k}$ has the form

$$
\mathbf{X}_{k}=\left[\begin{array}{c} 
\\
\mathbf{X}_{n-1} \\
\mathbf{0} \\
\vdots \\
\mathbf{0} \\
\mathbf{0}
\end{array}\right]+\left[\begin{array}{cccc}
\mathbf{I} \alpha_{0} & \mathbf{0} & \cdots & \mathbf{0} \\
\mathbf{I} \alpha_{1} & \mathbf{I} \alpha_{0} & \cdots & \mathbf{0} \\
\vdots & \vdots & & \vdots \\
\mathbf{I} \alpha_{n-1} & \mathbf{I} \alpha_{n-2} & & \mathbf{0} \\
-\mathbf{I} & \mathbf{I} \alpha_{n-1} & \cdots & \mathbf{0} \\
\mathbf{0} & -\mathbf{I} & \cdots & \mathbf{I} \alpha_{0} \\
\vdots & \vdots & & \vdots \\
\mathbf{0} & \mathbf{0} & \cdots & -\mathbf{I}
\end{array}\right]\left[\begin{array}{c}
\gamma_{n} \\
\vdots \\
\gamma_{k}
\end{array}\right]
$$


where $\mathbf{X}_{n-1}$ is a solution of equation (5) for $k=n-1$. So, the first term of this equation is a particular solution, while the second is an element of $\operatorname{ker}\left(\operatorname{Cont}^{k}(\mathbf{A}, \mathbf{B})\right)$. It can be separated into two simultaneous equations:

$$
\begin{aligned}
& \mathbf{X}_{k}^{1}=\left[\mathbf{X}_{n-1}\right]+\left[\begin{array}{cccc}
\mathbf{I} \alpha_{0} & \mathbf{0} & \cdots & \mathbf{0} \\
\mathbf{I} \alpha_{1} & \mathbf{I} \alpha_{0} & \cdots & \mathbf{0} \\
\vdots & \vdots & & \vdots \\
\mathbf{I} \alpha_{n-1} & \mathbf{I} \alpha_{n-2} & \cdots & \mathbf{I} \alpha_{0}
\end{array}\right]\left[\begin{array}{c}
\gamma_{n} \\
\vdots \\
\gamma_{2 n-1}
\end{array}\right] \\
& \mathbf{X}_{k}^{2}=\left[\begin{array}{ccccccc}
-\mathbf{I} \mathbf{I} \alpha_{n-1} & \cdots & \mathbf{I} \alpha_{0} & \mathbf{0} & \cdots & \mathbf{0} \\
\mathbf{0} & -\mathbf{I} & \mathbf{I} \alpha_{n-1} & \cdots & \mathbf{I} \alpha_{0} & \cdots & \vdots \\
\vdots & & & & & & \vdots \\
\mathbf{0} & \cdots & \cdots & \cdots & \cdots & \mathbf{0} & -\mathbf{I}
\end{array}\right]\left[\begin{array}{c}
\gamma_{n} \\
\vdots \\
\gamma_{k}
\end{array}\right]
\end{aligned}
$$

where $\mathbf{X}_{k}=\left[\mathbf{X}_{k}^{1} \mathbf{X}_{k}^{2}\right]^{T}$. Therefore, for any solution $\mathbf{X}_{k}$ of equation (5) there must exist $\mathbf{X}_{n-1}$ and $\left[\gamma_{n} \ldots \gamma_{k}\right]^{T}$ that fulfill equations (6) and (7).

Now, suppose that $\exists \mathbf{X}_{k}$ that fulfills equation (5) for $k=2 n-1$, but $\mathbf{X}_{k} \not \leq \mathbf{0}$. (it is easy to demonstrate, via Calley-Hamilton's theorem, that if there does not exist such solution, then there does not exist any solution $\mathbf{X}_{k}$ for any index $k \neq$ $2 n-1)$. Proceeding by contradiction, suppose also that for some $k>2 n-1$ there exists $\mathbf{X}_{k}^{\prime} \leq \mathbf{0}$ that fulfills equation (5) (if there exists a solution for $k<2 n-1$, then there also exists a solution for any $k \geq 2 n-1$, so, we only consider the case in which there exist solution for $k>2 n-1)$. So, there exist $\mathbf{X}_{n-1}^{\prime}$ and $\left[\gamma_{n}^{\prime} \cdots \gamma_{k}^{\prime}\right]^{T}$ that fulfill equations (6) and (7). Moreover, according to equation (7), it is easy to see, considering that $\hat{\mathbf{a}} \geq \mathbf{0}, \mathbf{X}_{k}^{\prime} \leq \mathbf{0}$ and following a backward substitution reasoning, that $\left[\gamma_{n}^{\prime} \cdots \gamma_{k}^{\prime}\right]^{T} \geq \mathbf{0}$. But, by the contradiction hypothesis, there do not exist $\left[\mathbf{X}_{n-1}\right]$ and $\left[\gamma_{n} \cdots \gamma_{2 n-1}\right]^{T}$ such that

$$
\begin{aligned}
{\left[\mathbf{X}_{n-1}\right]+} & {\left[\begin{array}{cccc}
\mathbf{I} \alpha_{0} & \mathbf{0} & \cdots & \mathbf{0} \\
\mathbf{I} \alpha_{1} & \mathbf{I} \alpha_{0} & \cdots & \mathbf{0} \\
\vdots & \vdots & & \vdots \\
\mathbf{I} \alpha_{n-1} & \mathbf{I} \alpha_{n-2} & \cdots & \mathbf{I} \alpha_{0}
\end{array}\right]\left[\begin{array}{c}
\gamma_{n} \\
\vdots \\
\gamma_{2 n-1}
\end{array}\right] \leq \mathbf{0} } \\
& {\left[\begin{array}{cccc}
-\mathbf{I} & \mathbf{I} \alpha_{n-1} & \cdots & \mathbf{I} \alpha_{1} \\
\mathbf{0} & -\mathbf{I} & \cdots & \mathbf{I} \alpha_{2} \\
\vdots & \vdots & & \vdots \\
\mathbf{0} & \cdots & \cdots & -\mathbf{I}
\end{array}\right]\left[\begin{array}{c}
\gamma_{n} \\
\vdots \\
\gamma_{2 n-1}
\end{array}\right] \leq \mathbf{0} }
\end{aligned}
$$

This is a contradiction, since the previous values of $\mathbf{X}_{n-1}^{\prime}$ and $\left[\gamma_{n}^{\prime} \cdots \gamma_{2 n-1}^{\prime}\right]^{T} \geq$ $\mathbf{0}$ clearly fulfill these last inequalities. 